\title{
The role of processing difficulty in the predictive utility of working memory span
}

\author{
MICHAEL BUNTING \\ University of Illinois, Chicago, llinois \\ and University of Missouri, Columbia, Missouri
}

\begin{abstract}
Storage-plus-processing working memory span tasks (e.g., operation span [OSPAN]) are strong predictors of higher order cognition, including general fluid intelligence. This is due, in part, to the difficulty of the processing component. When the processing component prevents only articulatory rehearsal, but not executive attentional control, the predictive utility is attenuated. Participants in one experiment $(N=59)$ completed Raven's Advanced Progressive Matrices (RAPM) and multiple versions of OSPAN and probed recall (PR). A distractor task (high or low difficulty) was added to PR, and OSPAN's processing component was manipulated for difficulty. OSPAN and PR correlated with RAPM when the processing component took executive attentional control. These results are suggestive of resource sharing between processing and storage.
\end{abstract}

Immediate memory tests are differentiable between those requiring storage and those requiring storage plus some form of additional processing. Storage-plusprocessing span tasks (e.g., reading span and operation span [OSPAN]) require high-level executive control (e.g., reading comprehension and mental calculation) and are widely regarded as useful measures of individual differences in working memory (WM) capacity, or one's WM span. They are, among other things, strong predictors of general fluid intelligence (see Conway, Cowan, Bunting, Therriault, \& Minkoff, 2002, for further elaboration). It remains unclear whether their predictive utility is due, in part, to the nature of the processing and its attentional demands or just to the sheer distraction that the processing component offers. The objective of this study was to examine this question and to determine whether dual-component span tasks are necessary to get predictive utility out of WM measures.

Daneman and Carpenter (1980; see also Broadbent, 1958; Just \& Carpenter, 1992) have suggested that process-

This research was completed in partial fulfillment of the requirements for the degree of Doctor of Philosophy at the University of IIlinois at Chicago. M.B. was supported by a dissertation fellowship from the University of Illinois at Chicago while these experiments were being conducted and by a postdoctoral fellowship at the University of Missouri-Columbia from the Missouri Rehabilitation Research Training Program (Kristofer Hagglund, P.I.), by the National Institute of Child Health and Human Development, and by National Institutes of Health Grant 2 T32 HD07460-09 while this article was being prepared. I thank Andy Conway, Nelson Cowan, and Tim Miura for comments on drafts of the manuscript. I also thank the dissertation committee members, Andy Conway, Mike Kane, Jim Pellegrino, Keith Thiede, and Jenny Wiley, and undergraduate assistants Phil Cantore, Mike Hanna, and Ed Sandoval for help testing participants. Correspondence concerning this article should be addressed to M. Bunting, Center for Advanced Study of Language, University of Maryland, P.O. Box 25, College Park, MD 65211 (e-mail: mbunting@casl.umd.edu). ing and storage are inextricably linked in their competition for a single, flexible facility of limited capacity, and they hypothesized that more efficient processing permits more room for storage. Conway and Engle (1996) have proposed a similar sort of resource sharing for their OSPAN WM task, suggesting that it requires a perpetual trade-off between performing an operation and encoding a word until recall is prompted. In OSPAN, participants read aloud and verify the accuracy of mathematical equations (the processing component) while trying to remember some memoranda for later recall (the storage component).

Processing load (i.e., the numbers of operations and new representations created for accomplishing the processing) of distracting tasks has been varied before (Barrouillet, Bernardin, \& Camos, 2004; Saito \& Miyake, 2004; Waters \& Caplan, 1996), but seldom in a direct test of the effect of varying processing load on the predictive utility of WM span. Conway and Engle (1996) showed that the difficulty of the math problems (addition and subtraction vs. multiplication and division, but also the number of intervening operations) does not matter per se; individual WM spans (the number of words recalled in OSPAN) were the same with easy and difficult math problems. Critically, Conway and Engle's difficulty manipulation had no impact on the predictive utility of OSPAN. They manipulated the difficulty of the operations and, in doing so, manipulated the processing load. Their easy problems had fewer intervening operations than did the difficult problems (e.g., $7+9$ vs. $2 \times 8-5$ ), but even singledigit arithmetic problems require central executive resources (DeStefano \& LeFevre, 2004). All of Conway and Engle's problems had some processing load and required some effort; hence, processing was never entirely automatic. Essentially, Conway and Engle had a null effect, because all levels of difficulty yielded span scores that were equally predictive.

The predictive utility of tasks such as OSPAN could be due to the demand for central executive resources during 
interweaving processing-storage activities. Processing load is arguably larger in complex than in simple span tasks. Concurrent verbal processing undoubtedly interferes with maintenance rehearsal in the articulatory loop. Furthermore, with increased complexity, the processing component may also demand controlled (executive) attention. This would be consistent with Engle and colleagues' controlled attention view of WM, which attributes individual differences in WM capacity to an executive attention control mechanism that combats some form of salient interference (e.g., proactive interference, response competition, or habitual but inappropriate responses; Bunting, 2006; Engle \& Kane, 2004).

The present study combined experimental and differential methodologies to show that processing load affects mean task performance concomitantly with predictive utility. This experiment manipulated the ease of processing so as to have minimal processing load while still preventing overt rehearsal (i.e., the processing component was more automatized than that in Conway \& Engle, 1996). The memory tasks were OSPAN (a storage-plus-processing task) and probed recall (PR; a storage-only task), and the criterion task was Raven's Advanced Progressive Matrices (RAPM). OSPAN processing difficulty was minimized in a way that removed the demand of solving math equations, while still preventing rehearsal. PR was made more difficult with the addition of easy and difficult distractor tasks. Varying processing load should affect the well-documented relationship between WM (measured by OSPAN and PR) and fluid intelligence (measured by RAPM) to the extent that processing load is a factor in WM span scores (Conway et al., 2002).

The predictions were twofold. Relieving the processing demands on OSPAN should improve scores but attenuate the OSPAN-RAPM relationship. Conversely, increasing the processing demands on PR should hurt scores but also should make early list items predictive of RAPM in proportion to the attentional demands of the distractor task. Hence, this would show that storage-plus-processing tasks have predictive utility when they force the maintenance of information while the central executive is recruited to concurrently perform some other task.

\section{METHOD}

\section{Participants}

Fifty-nine ( 35 of them female) native English-speaking University of Illinois-Chicago undergraduates received course credit. Three additional participants were excluded for not completing all the tasks.

\section{Tasks and Procedure}

The participants were individually tested in 1-h sessions on the same day and time for 2 consecutive weeks. Session 1 had the PR tasks and the OSPAN experimental task, counterbalanced for order. Session 2 had the RAPM task. The OSPAN control task was administered separately as part of another experiment, which 41 participants completed first (the remaining participants completed the present experiment first).

Operation span (adapted from Conway et al., 2002). The participants completed a control (i.e., typical) version of OSPAN and an easier, experimental version that minimized processing difficulty. In each trial of both versions, an equation and a one-syllable, high-frequency word memorandum were visually presented. The equations began with multiplication or division of two integers from 1 to 10 , and a third integer from 1 to 9 was added to or subtracted from the result. Solutions were correct half of the time. False solutions were correct solutions plus 2 . The participants read the equation aloud at trial onset, said "yes" or "no" to verify its accuracy, and read aloud and remembered the word. To prevent rehearsal, the experimenter immediately initiated the next trial by keypress. After multiple trials, a cue ("???") prompted written serial recall of the memoranda. Twelve sets of trials ( 3 of each set size from two to five items) were presented in random order. In the control task, a unique mathematical operation appeared with each memorandum. In the experimental version, the same mathematical operation (different for each participant) appeared with each memorandum but was read aloud and at the same pace each time. The response sheet had two blanks for each of 3 practice sets and six blanks for each of 12 test sets. Table 1 shows examples of the operations and memoranda for a five-item set.

Probed recall (adapted from Cohen \& Sandberg, 1977). In each trial, 12 serial memoranda (randomized digits from the set $0-9$ ) were visually presented at 1 digit/sec. A letter cue $(A, B$, or $C)$ lasting $2 \mathrm{sec}$ prompted the written serial recall of the first, middle, or final four memoranda, respectively. The restrictions on randomization were that throughout each list, (1) each digit was used at least once, (2) no digit was repeated more than twice, (3) no two digits appeared in forward numerical order, and (4) a digit was never repeated within the primacy, middle, or recency groups. Each task had 3 practice and 24 test trials (A, B, and C groups were cued equally). The participants initiated trials by keypress, which began with a 1-sec centered fixation ( + ). An audible tone signaled the end of the 10 -sec response window that began with the onset of the letter cue, and a ready message was displayed on-screen until the participant initiated the next trial. The response sheet had four blanks per trial.

The participants completed three versions of PR, including (1) control (no dual task), (2) PR with articulatory suppression (AS), and (3) PR with random letter generation (RLG). Typically in PR, later list items (i.e., recency items) fail to benefit from rehearsal and, therefore, measure WM well (Cohen \& Sandberg, 1977). Early list items (i.e., primacy items) typically do not measure WM span well, probably because maintenance rehearsal buffers these items. Consequently, individual differences are attributable to rehearsal efficiency (e.g., articulation rate), rather than to executive control.

Table 1

Examples of the Operations and Memoranda for a

Five-Item Trial in Operation Span With Constant-Processing or Varied-Processing Components

\begin{tabular}{cccccc} 
& \multicolumn{2}{c}{ Constant Processing } & & \multicolumn{2}{c}{ Varied Processing } \\
\cline { 2 - 3 } \cline { 5 - 6 } Item No. & Operations & Memoranda & & Operations & Memoranda \\
\hline 1 & $(9 \times 3)+2=29$ & lean & & $(8 \times 4)-5=29$ & knife \\
2 & $(9 \times 3)+2=29$ & share & & $(6 \times 3)-2=18$ & set \\
3 & $(9 \times 3)+2=29$ & worth & & $(10 \div 2)-1=4$ & fair \\
4 & $(9 \times 3)+2=29$ & act & & $(6 \times 3)+2=20$ & dress \\
5 & $(9 \times 3)+2=29$ & wire & & $(9 \div 3)-2=1$ & heat \\
\hline
\end{tabular}


AS and RLG were added to PR for the purpose of preventing rehearsal and potentially implicating WM in the maintenance of early list items. Baddeley (2000) called AS a "low processing" distractor task. Articulation, repeating "the" once per digit in PR, began at trial onset and stopped when the recall cue appeared.

Concurrent RLG, the "high processing" distractor task, was adapted from Baddeley (1966). The participants articulated one letter per two digits in PR.' The digits were shown in alternating colors (red and black), and red digits prompted RLG. (Note that for consistency, the digits were also presented in alternating colors in the PR-control and PR-AS tasks, but the participants ignored this color change.) As instructed, the participants avoided generating letters in forward alphabetical order (e.g., A-B-C) and repeating the same letter more often than would be expected by chance. The experimenter tracked responses on RLG.

The participants practiced RLG for eight trials of 12 symbols each before the real test. Two alternating symbols, forward (" $/$ ") and backward (")") slashes, were presented in alternating colors at the rate of $1 \mathrm{symbol} / \mathrm{sec}$ in place of digits in the real test. The red color signaled RLG.

RAPM (see Raven, Raven, \& Court, 1998). RAPM presented diagrammatic puzzles exhibiting serial, bidirectional change simultaneously. The participants chose a missing piece from an eightchoice selection. The practice, administered for $5 \mathrm{~min}$, had 12 items. The test, administered in $40 \mathrm{~min}$, had 36 items.

\section{Scoring}

Operation span and probed recall. Recall accuracy was tabulated for each OSPAN and for primacy, middle, and recency items on each PR task. One point was awarded per correct item in the correct serial position, and this sum, divided by the possible score, yielded the total score. As is typical in OSPAN, equations responded to incorrectly were not factored into scores, but participants below an $85 \%$ accuracy criterion were excluded.

Two measures of randomness were computed for RLG. One measure, redundancy, was the standard deviation of the mean number of times each letter of the alphabet was used. If perfectly random, each letter would be generated equally often with zero standard deviation; greater deviation from zero reflects greater nonrandomness. A second measure, randomness, was the number of two-letter alphabetical sequences per trial (e.g., A-B), which were summed across trials.

RAPM. The score was the sum of correct responses to the items in the test. The practice set was not analyzed.

\section{RESULTS}

Table 2 displays means, standard deviations, reliability (Cronbach's alpha), skewness, and kurtosis for all the scores. The assumption of normality was not violated; estimates of skewness and kurtosis were well within the accepted limits of skewness (below 3 ) and kurtosis (below 4) (Kline, 2004). Ceiling and floor effects were unapparent, since all the means were more than $1.0 S D$ from their respective minimum and maximum. Mahalanobis distance did not detect multivariate outliers that would have fundamentally changed the experimental effects or patterns of correlations.

\section{Operation Span}

Everyone met the $85 \%$ accuracy criterion for operations. Span scores were higher in OSPAN-experimental than in OSPAN-control $[t(58)=4.22, p<.001]$. The correlation between OSPAN-control and RAPM was significant and consistent in magnitude with published reports (Conway et al., 2002). Because only the reliable portions of two sets of scores can be correlated, the unreliable portions are random error and uncorrelated. Consequently, the magnitude of the correlation between any two scores is limited (attenuated) by their respective unreliability. The correction for attenuation offsets this discrepancy. Following Schmidt and Hunter's (1999) recommendations, I will report the raw and corrected forms of the correlations (see

Table 2

Means, Standard Deviations, Reliability (a), Skewness, and Kurtosis for Scores on Each Version of the Operation Span, Probed Recall, and Raven's Advanced Progressive Matrices Tasks $(N=59)$

\begin{tabular}{|c|c|c|c|c|c|}
\hline Score & $M$ & $S D$ & $a$ & Skewness & Kurtosis \\
\hline \multicolumn{6}{|c|}{ Operation Span } \\
\hline \multicolumn{6}{|l|}{ Control (varied processing) } \\
\hline Mean proportion correct & .68 & .16 & .76 & -0.12 & -0.28 \\
\hline \multicolumn{6}{|c|}{ Experimental (constant processing) } \\
\hline Mean proportion correct & .75 & .12 & .64 & 0.14 & 0.07 \\
\hline \multicolumn{6}{|c|}{ Probed Recall } \\
\hline \multicolumn{6}{|l|}{ Control } \\
\hline Primacy & .47 & .21 & .70 & 0.35 & -0.84 \\
\hline Middle & .38 & .19 & .63 & -0.05 & -0.54 \\
\hline Recency & .75 & .19 & .73 & -0.68 & -0.54 \\
\hline \multicolumn{6}{|l|}{ + Articulatory suppression } \\
\hline Primacy & .30 & .16 & .64 & 0.67 & -0.37 \\
\hline Middle & .28 & .16 & .59 & 0.97 & 0.66 \\
\hline Recency & .36 & .13 & .65 & 0.06 & -0.03 \\
\hline \multicolumn{6}{|l|}{ + Random letter generation } \\
\hline Primacy & .13 & .08 & .65 & 0.39 & -0.65 \\
\hline Middle & .10 & .06 & .60 & 0.26 & $-1.15^{*}$ \\
\hline Recency & .19 & .12 & .67 & 0.64 & -0.04 \\
\hline
\end{tabular}

Raven's Advanced Progressive Matrices Test

\begin{tabular}{llllll} 
Mean accuracy & 21.98 & 5.01 & 0.31 & -0.28 \\
\hline
\end{tabular}

$p<.01$. 
Bunting, 2006, for a similar method of analysis). Figure 1 shows the correlations, corrected for attenuation, for both versions of the OSPAN task (raw correlations are reported in the figure caption).

Did holding the operation constant also produce a meaningful change in the correlation between OSPAN scores and RAPM? Two comparisons seem relevant: (1) a direct test of the difference between the OSPAN-RAPM correlations on the experimental and control tasks and (2) a test of whether each score in the experimental task accounts for similar variance in RAPM.

By Steiger's (1980) test for dependent correlations, the difference between the raw correlations for the experimental $(r=.32)$ and the control $(r=.41)$ tasks was not significant $(z<1$, n.s.). When corrected for attenuation, the difference between the correlations for the experimental $(r=.39)$ and the control $(r=.47)$ tasks approached significance $(z=1.45, p=.07)$.

Sequential multiple regression tested whether the OSPAN-RAPM correlations are of similar magnitude and whether each OSPAN task accounted for similar (i.e., homogeneous) variance in RAPM. The experimental task alone accounted for significant variance at Step $1\left[R^{2}=\right.$ $.10 ; F(1,57)=6.53, p<.01]$. The control task accounted for additional variance in RAPM when added at Step 2

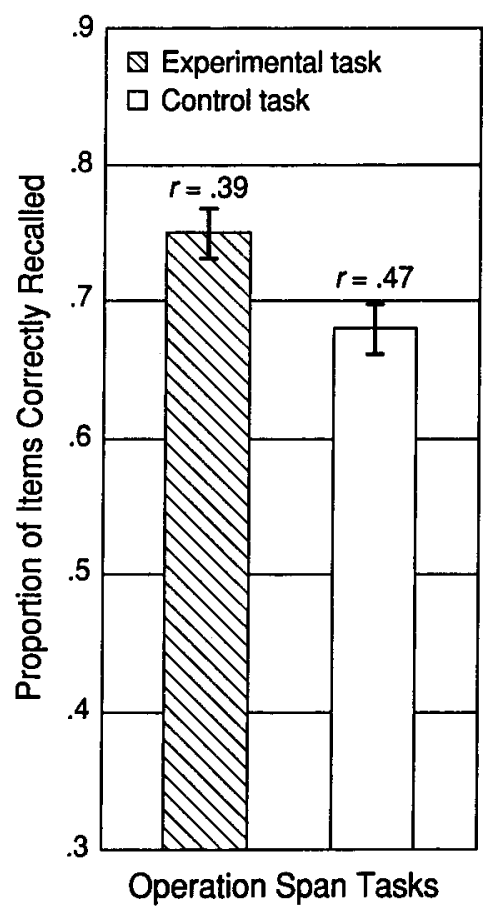

Figure 1. The mean proportions correct on the control and experimental versions of the operation span task with constantprocessing or varied-processing components, respectively. Bars represent the standard errors of the means. The correlations, which are corrected for attenuation (no significance test available), are between operation span scores and performance on Raven's advanced progressive matrices. The uncorrected correlations for the experimental and control tasks are $.32^{*}$ and .41 *, respectively, where an asterisk indicates that $p<.05$.
$\left[\Delta R^{2}=.07 ; \Delta F(1,56)=4.83, p<.03\right]$. However, when conducted in the reverse with the control task entered first, the experimental task failed to capture additional variance in RAPM [for Step $1, R^{2}=.17 ; F(1,57)=11.37, p<$ .001 ; for Step $2, \Delta R^{2}<.01 ; \Delta F(1,56)<1$, n.s.].

\section{Probed Recall}

Did the addition of either distractor task reduce accuracy on primacy, middle, or recency items? Scores should be highest for the control task without distraction and lowest for the PR task with RLG, the difficult distractor. The repeated measures ANOVA for task type (control, AS, and RLG) $\times$ cue (primacy, middle, and recency) yielded a significant interaction $\left[F(4,232)=30.7, M S_{\mathrm{e}}=0.015\right.$, $p<.001]$. Figure 2 shows the mean proportion correct for primacy, middle, and recency items by task and the correlation, corrected for attenuation, between each of these items and RAPM (raw correlations are reported in the figure caption). Main effects for task type and cue were also significant $\left[F(2,116)=275.5, M S_{\mathrm{e}}=0.024\right.$, $p<.001$, and $F(2,116)=83.4, M S_{\mathrm{e}}=0.016, p<.001$, respectively]. The Appendix lists the RLG analysis of randomness.

The distractor tasks hurt performance on the primary task, more so for RLG. This is evident in the simple effect of task type at each level of cue $\left[F(2,116)=94.79, M S_{\mathrm{e}}=\right.$ $0.019, p<.017 ; F(2,116)=57.14, M S_{\mathrm{e}}=0.019, p<$ .017 ; and $F(2,116)=286.89, M S_{\mathrm{e}}=0.017, p<.017$, for primacy, middle, and recency items, respectively; a Bonferroni correction was applied]. Pairwise comparisons confirmed that memory for primacy, middle, and recency items was significantly better in the control task than when AS was added, which, in turn, was better than when RLG was added.

For the PR-control and PR-AS tasks, middle and recency scores, but not primacy scores, significantly correlated with RAPM (see Figure 2). As was hypothesized, recency items in both tasks significantly correlated with RAPM, but even with AS preventing rehearsal, primacy items in PR-AS failed to correlate significantly with RAPM.

The results were different for scores on PR-RLG where recency and primacy items significantly correlated with RAPM. Middle items did not. ${ }^{2}$ As for OSPAN, two comparisons seem relevant: (1) a direct test of the difference between the relevant PR-RAPM correlations and (2) a test of whether scores on the experimental tasks accounted for similar variance in RAPM.

By Steiger's (1980) test for dependent correlations, the correlation between primacy items in PR-RLG and $\operatorname{RAPM}(r=.43)$ significantly differed from the correlation between primacy items in PR-control and RAPM $(r=.19)$, but not from the correlation between primacy items in PR-AS and RAPM $(r=.25 ; z=1.55, p=.05$, and $z=1.12$, n.s., respectively; one-tailed). When the correlations are corrected for attenuation, both analyses are significant $(z=3.34, p<.001$, and $z=2.50, p<.01$, respectively; one-tailed). Correlations between primacy items in PR-control and RAPM and primacy items in 


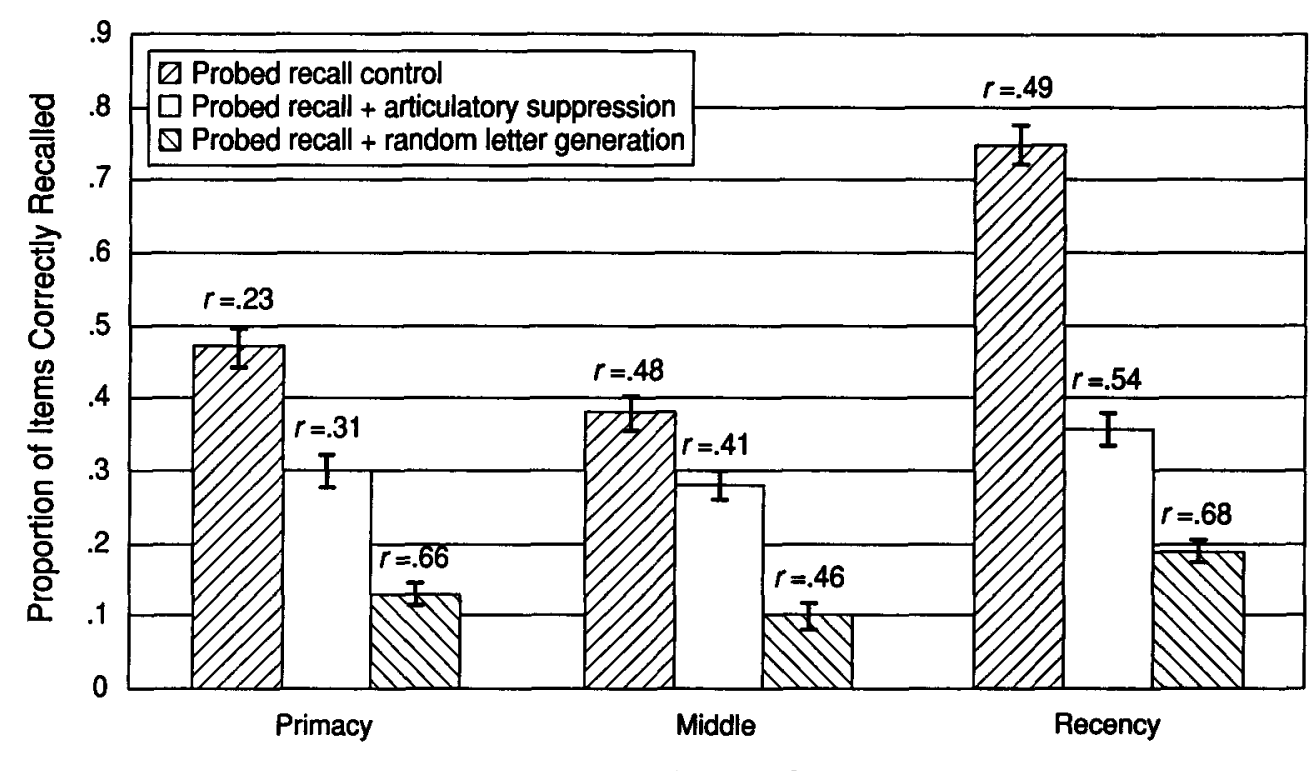

Partial Recall Cue

Figure 2. The mean proportions correct on primacy, middle, and recency items in three versions of probed recall (control, probed recall with articulatory suppression, and probed recall with random letter generation). Bars represent the standard errors of the means. The correlations, which are corrected for attenuation (no significance test available), are between those for the probed recall scores and those for performance on Raven's advanced progressive matrices. The uncorrected correlations for the probed recall control are, from left to right, $.19, .39^{\star}$, and $.40^{\star}$; for probed recall with articulatory suppression, they are $.25, .31^{\star}$, and $.43^{\star}$; and for probed recall with random letter generation, they are $.43^{\star}, .21$, and $.46^{\star}$, where an asterisk indicates that $p<.05$.

PR-AS differed neither before nor after correcting for attenuation $(z<1.0$, n.s., and $z<1.0$, n.s., respectively).

As was hypothesized, primacy items in PR-RLG significantly predicted RAPM, whereas the PR-control did not. In confirmation of this, sequential multiple regression indicated that primacy items in PR-RLG accounted for a significant portion of the variance in RAPM when entered alone at Step $1\left[R^{2}=.19 ; F(1,57)=12.99, p<.001\right]$. At Step 2, primacy items in PR-control did not account for more unique variance $\left[\Delta R^{2}=.02 ; \Delta F(1,56)=1.10\right.$, n.s.]. For the analysis in the reverse direction, primacy items in PR-control still failed to capture any variance in RAPM when added alone at Step $1\left[R^{2}=.03 ; F(1,57)=\right.$ 2.10 , n.s.]. But primacy items from PR-RLG did at Step 2 $\left[\Delta R^{2}<.20 ; \Delta F(1,56)=11.69, p<.001\right]$.

Primacy items in PR-RLG not only correlated with RAPM, but also loaded on the same factor as recency items from all three tests in an exploratory factor analysis with varimax rotation. Table 3 depicts the component factor matrix. Primacy and recency items from each version of $P R$ reduced to two factors with eigenvalues greater than 1.0. Primacy items from PR-control and PR-AS loaded on one factor, whereas the remaining items loaded on a second factor. Scores from the control and experimental versions of OSPAN were also included and loaded on the same factor as recency items from PR, which indicates that the experimental version of OSPAN behaved more like a standard WM measure than was expected. Therefore, much as was expected, the components of the PR tasks that behaved most like OSPAN, a standard WM measure, were the PR recency items and the PR primacy items with some level of executive involvement (PR-RLG).

\section{DISCUSSION}

These experimental effects suggest that the predictive utility of WM measures, such as OSPAN, is based on a resource trade-off between processing and storage. With a single equation for all the items, OSPAN acted like AS and posed less demand on attention than did actually solving equations, as the increased score reflected. Conversely, increasing the competing attentional demands during PR

Table 3

The Component Matrix for the Exploratory Factor Analysis With Varimax Rotation of Scores for Primacy and Recency Items in Each Version of the Probed Recall Task and Scores for the Control and Experimental Versions of the Operation Span Task

\begin{tabular}{lccc}
\hline & \multicolumn{2}{c}{ Component } \\
\cline { 2 - 3 } \multicolumn{1}{c}{ Items } & 1 & 2 \\
\hline Operation span varied processing (control) & .811 & .247 \\
Operation span constant processing (experimental) & .755 & .181 \\
Probed recall control (recency) & .634 & .149 \\
Probed recall + articulatory suppression (recency) & .789 & .205 \\
Probed recall + random letter generation (primacy) & .435 & .106 \\
Probed recall + random letter generation (recency) & .664 & .146 \\
Probed recall control (primacy) & .257 & .674 \\
Probed recall + articulatory suppression (primacy) & .127 & .879 \\
\hline
\end{tabular}


made the primary task more difficult to perform. As is consistent with resource-sharing hypotheses, there was increasingly less room for storage as processing demands increased with AS and then RLG.

Competing attentional demands during encoding were critical to the predictive utility of these measures. The OSPAN-RAPM correlation was attenuated when a single equation was used for all the items, as was indicated by the regression analysis, but not as much as was expected, as was indicated by the correlation difference test. Conversely, adding distraction during PR brought out a definite primacy-item-RAPM correlation, but only when the attentional demands were high with RLG.

Manipulating processing load can inadvertently affect processing time (i.e., less processing takes less time), which makes it difficult for researchers to do. To avoid this problem, articulation rate was held constant in the OSPAN experimental task. In PR-RLG, the participants engaged in RLG on every other digit, not on every digit, as in PR-AS. The critical result for PR-RLG was not a matter of RLG's requiring more processing time; rather, it required more attention. The predictive utility of $P R-R L G$ was due to the attentional complexity of the distractor task, and not just to rehearsal prevention.

These effects are consistent with the results in Barrouillet et al. (2004, Experiment 2), who developed three versions of OSPAN. Their continuous OSPAN was akin to traditional OSPAN and the control task in the present experiment, but each element of the operation string was presented individually and at a computer-controlled pace. For example, the participants encountered small operations in successive order (e.g., $4 /+1 /-2$ ) and solved them as they were shown, saying, "four," "plus one, five," "minus two, three." In their baba span, the participants spoke the syllable "ba" in place of each small operation above. In their reading operation span (akin to the experimental OSPAN in the present experiment), the participants read operations and intermediate solutions aloud (e.g., " $4 /+1 / 5 /-2 / 3$ "), rather than solving them.

Barrouillet et al.'s (2004) OSPAN tasks were matched for the length of processing, thus ruling out a strictly interpreted task-switching hypothesis (cf. Towse, Hitch, \& Hutton, 2000) when they found that scores were lowest on the continuous operations task, higher on the reading operations task, and highest on the baba span task. Task switching would attribute higher span scores to more time spent on the processing component and forgetting from decay. This was the case neither in Barrouillet et al. nor here, supporting a resource-sharing hypothesis. Unlike in the present experiment, Barrouillet et al. did not test the effect of their manipulations on the predictive utility of their span tasks. However, in a follow-up study, Lépine, Barrouillet, and Camos (2005) found that timeconstrained WM span tasks with simple processing (reading letters while remembering numbers) or complex processing (continuous OSPAN) had higher predictive power than did traditional WM span tasks that were participant paced. Their results and the results of the present experi- ment conjointly point to the common theme that sustained attentional demands on processing, not the time spent on it or the degree of difficulty per se, determine the predictive utility of complex, dual-task measures. Simple AS does not impose the sort of demand encountered in RLG (the present experiment) or carefully time-constrained letter reading (Lépine et al., 2005).

Finally, these data, as well as those from Barrouillet et al.'s (2004) experiments, fit Cowan's (2001) concept of attention as a flexible mental resource. By Cowan's view, the capacity-limited focus of attention operates as an adjustable spotlight that might zoom in to maintain a goal in the face of distraction or zoom out to apprehend multiple items up to the capacity of attention. Recency items in the PR-control task might work like the latter; attention zooms out to apprehend the most recently presented items. Attention can serve goal maintenance in the presence of complex distractors (RNG or complex math operations), but only at a cost to the use of attention for temporary storage. Simple AS, although detrimental to overt rehearsal, does not obviate attention in any consistent way. Future research should look for new ways to investigate the basis of storage-processing trade-offs in WM.

Although this discussion has focused on the change in ease of processing when OSPAN uses one equation for all items, another explanation is that using one equation creates fewer interfering memory representations. The same may be true for AS versus RLG. To test this in future research, consider an AS experiment in which a different word is articulated on each trial, thus increasing the number of irrelevant memory representations, but not necessarily the processing difficulty.

The measurement of WM capacity has implications for theories of WM, fluid intelligence, and cognitive abilities, including, but not limited to, language and reading comprehension and learning and skill acquisition. Processing difficulty is important to dual-component WM tasks and individual differences in WM.

\section{REFERENCES}

BADDELEY, A. D. (1966). The capacity for generating information by randomization. Quarterly Journal of Experimental Psychology, 18, 119-129.

BADDELEY, A. D. (2000). Short-term and working memory. In E. Tulving \& F. I. M. Craik (Eds.), The Oxford handbook of memory (pp. 77-92). New York: Oxford University Press.

Barrouillet, P., Bernardin, S., \& Camos, V. (2004). Time constraints and resource sharing in adults' working memory spans. Journal of Experimental Psychology: General, 133, 83-100.

Broadbent, D. E. (1958). Perception and communication. New York: Pergamon.

BUNTING, M. (2006). Proactive interference and item similarity in working memory. Journal of Experimental Psychology: Learning, Memory, \& Cognition, 32, 183-196.

Cantor, J., Engle, R. W., \& Hamilton, G. (1991). Short-term memory, working memory, and verbal abilities: How do they relate? Intelligence, 15, 229-246.

COHEN, R. L., \& SANDBERG, T. (1977). Relation between intelligence and short-term memory. Cognitive Psychology, 9, 534-554.

Conway, A. R. A., Cowan, N., Bunting, M. F., Therriault, D. J., 
\& MinKoFF, S. R. B. (2002). A latent variable analysis of working memory capacity, short-term memory capacity, processing speed, and general fluid intelligence. Intelligence, 30, 163-183.

Conway, A. R. A., \& ENGLE, R. W. (1996). Individual differences in working memory capacity: More evidence for a general capacity theory. Memory, 4, 577-590.

Cowan, N. (2001). The magical number 4 in short-term memory: A reconsideration of mental storage capacity. Behavioral \& Brain Sciences, 24, 87-114.

Daneman, M., \& CARPENTER, P. A. (1980). Individual differences in working memory and reading. Journal of Verbal Learning \& Verbal Behavior, 19, 450-466.

DEStEFANO, D., \& LeFEVRE, J.-A. (2004). The role of working memory in mental arithmetic. European Journal of Cognitive Psychology, 16, 353-386.

ENGLE, R. W., \& KANE, M. J. (2004). Executive attention, workingmemory capacity, and a two-factor theory of cognitive control. In B. H. Ross (Ed.), The psychology of learning and motivation: Advances in research and theory (Vol. 44, pp. 145-199). New York: Elsevier.

JUST, M. A., \& CARPENTER, P. A. (1992). A capacity theory of comprehension: Individual differences in working memory. Psychological Review, 99, 122-149.

KLINE, R. B. (2004). Principles and practice of structural equation modeling (2nd ed.). New York: Guilford.

LÉPINE, R., BARRoUILLET, P., \& CAMOS, V. (2005). What makes working memory spans so predictive of high-level cognition? Psychonomic Bulletin \& Review, 12, 165-170.
RaVen, J. C., RAVEN, J. E., \& CourT, J. H. (1998). Progressive matrices. Oxford: Oxford Psychologists Press.

SaIto, S., \& MiYAKE, A. (2004). On the nature of forgetting and the processing-storage relationship in reading span performance. Journal of Memory \& Language, 50, 425-443.

SCHMIDT, F. L., \& HUNTER, J. E. (1999). Theory testing and measurement error. Intelligence, 27, 183-198.

SteIGER, J. H. (1980). Tests for comparing elements of a correlation matrix. Psychological Bulletin, 87, 245-251.

TOWSE, J. N., HrTCh, G. J., \& HUTTON, U. (2000). On the interpretation of working memory span in adults. Memory \& Cognition, 28, 341-348.

WATERS, G. S., \& CAPLAN, D. (1996). The measurement of verbal working memory capacity and its relation to reading comprehension. Quarterly Journal of Experimental Psychology, 49A, 51-79.

\section{NOTES}

1. In piloting, generating random letters for every digit in probed recall proved too challenging.

2. Middle items were not germane to the hypotheses, in part because prior data, which showed middle item scores to be highly volatile, did not help me to make good a priori predictions. Cohen and Sandberg (1977, Experiment 1) reported widely ranging middle-item-IQ correlations that were significant for two of seven groups (correlations ranged from -.08 to $.52 ; M=.25, S D=.22$ ). Cantor, Engle, and Hamilton (1991) reported a similar pattern of modest but significant correlations between middle items and scores on the verbal portion of the Scholastic Aptitude Test.

\section{APPENDIX \\ Analysis of Randomness for Random Letter Generation}

Two measures of randomness were analyzed for RLG. At issue was whether the participants sacrificed randomness to improve scores on probed recall. Scores from the measure of redundancy ranged from 1.31 to 6.36 $(M=4.02, S D=1.11)$, where higher scores reflected less randomness. However, the measure of redundancy was unrelated to primacy, middle, or recency scores on probed recall with $\mathrm{RLG}(r=-.01, \mathrm{n} . \mathrm{s} ., r=.04, \mathrm{n} . \mathrm{s}$., and $r=-.03$, n.s., respectively). The mean number of alphabetical sequences was also low $(M=5.02)$, given the frequency with which alphabetical sequences of two letters could appear. This score was unrelated to primacy, middle, or recency scores $(r=.15$, n.s., $r=.13$, n.s., and $r=.12$, n.s., respectively).

(Manuscript received February 17, 2005;

revision accepted for publication April 21, 2006.) 